Anaesthesist 2014 · 63:5

DOI 10.1007/s00101-013-2271-x

Online publiziert: 9 . Januar 2014

c) Springer-Verlag Berlin Heidelberg 2014

\section{R. Klamroth}

Klinik für Innere Medizin - Angiologie, Hämostaseologie und Pneumologie, Hämophiliezentrum und Gerinnungssprechstunde, Vivantes Klinikum im Friedrichshain, Berlin

\section{Operation und Hämophilie}

\author{
Kein Problem bei gutem \\ interdisziplinären Management
}

Patienten mit hereditären Gerinnungsstörungen wie der Hämophilie stellen eine besondere Herausforderung für den betreuenden Arzt in der perioperativen Situation dar. Das liegt an der Seltenheit der Erkrankung - in Deutschland leben etwa 5000 Patienten mit einer Hämophilie A und 800 Patienten mit einer Hämophilie B - und der damit verbundenen Unsicherheit in notwendiger Diagnostik und Therapie.

Die funktionierende Gerinnung ist essenziell für jeden operativen Eingriff, sodass ohne adäquate Behandlung relevante Blutungskomplikationen bei Patienten mit einer Hämophilie zu erwarten sind.

Die Langzeitbetreuung dieser Patienten erfolgt in der Regel in einem spezialisierten hämostaseologischen Zentrum. Dort sind die Expertise und die Logistik für einen operativen Eingriff verfügbar. Trotzdem kann v. a. in der Notfallsituation jeder Arzt mit Hämophiliepatienten konfrontiert werden. Kenntnisse des Krankheitsbilds sowie der entsprechenden Diagnostik und Therapie sind dabei hilfreich.

Der Beitrag von Lison u. Spannagl in der vorliegenden Ausgabe von Der Anaesthesist widmet sich ausführlich dem perioperativen Management bei Patienten mit Hämophilie. Bei Hämophilie A ist der Gerinnungsfaktor VIII vermindert bzw. fehlt vollständig, bei Hämophilie B der Gerinnungsfaktor IX. Klinisch fallen diese Patienten primär durch Gelenk- und Muskelblutungen auf. Bei Verletzungen kann es zu massiven inneren und/oder äußeren Blutungen kommen.
Zwei Aspekte möchte ich aus Sicht des Hämostaseologen in der perioperativen Situation besonders hervorheben: Ein Grundpfeiler der Hämophilietherapie ist die Substitution des fehlenden Gerinnungsfaktors. Wichtigste Voraussetzung dafür ist die Verfügbarkeit des jeweiligen Gerinnungsfaktorenkonzentrats. Patienten sollten bei elektiven Eingriffen das Gerinnungsfaktorenkonzentrat erhalten, auf das sie eingestellt sind. In der Notfallsituation sollte auf das am schnellsten verfügbare Faktorenkonzentrat zurückgegriffen werden.

Der zweite Grundpfeiler ist die Überwachung der Substitutionstherapie. Der fehlende Gerinnungsfaktor sollte in den Normalbereich zum Zeitpunkt des operativen Eingriffs angehoben werden und muss, angepasst an die Größe des operativen Eingriffs und den Verlauf der Wundheilung, in der postoperativen Phase weitersubstituiert werden. Voraussetzung dafür ist die Verfügbarkeit einer zeitnahen Bestimmung des Faktors VIII bzw. des Faktors IX für das optimale Monitoring der Substitutionstherapie. Die regelmäßige Bestimmung des substituierten Gerinnungsfaktors ist notwendig, da die pharmakokinetischen Parameter wie Anstieg des Gerinnungsfaktors und die Halbwertszeit einer großen Schwankung zwischen verschiedenen Patienten unterliegen. Nur mit einer individuellen, laboradaptierten Dosierung können sowohl Unter- als auch Überdosierung vermieden werden.

Im Beitrag von Lison u. Spannagl wird ausführlich darauf eingegangen. Er vermittelt dem interessierten Leser das Rüst- zeug für ein erfolgreiches perioperatives Management von Patienten mit Hämophilie.

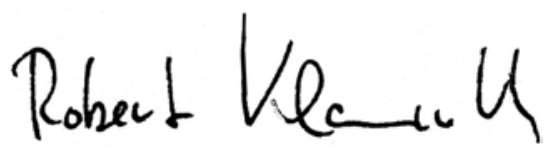

R. Klamroth

\section{Korrespondenzadresse}

\section{Dr. R. Klamroth}

Klinik für Innere Medizin - Angiologie, Hämostaseologie und Pneumologie, Hämophiliezentrum und Gerinnungssprechstunde,

Vivantes Klinikum im Friedrichshain Landsberger Allee 49, 10249 Berlin robert.klamroth@vivantes.de

\section{Einhaltung ethischer Richtlinien}

Interessenkonflikt. R. Klamroth gibt an, dass kein Interessenkonflikt besteht. 